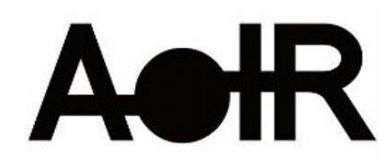

Selected Papers of \#AolR2021: The 22nd Annual Conference of the Association of Internet Researchers Virtual Event / 13-16 Oct 2021

\title{
Effects of Physical Distance and Social Closeness in Virtual Reality and Text on Spatial Distance Estimation
}

\author{
Andrea Stevenson Won, PhD \\ Cornell University \\ Byungdoo Kim, PhD \\ Cornell University \\ Introduction
}

The COVID-19 pandemic has imbued the phrases "social distancing" and "physical distancing" with new meaning. However, researchers have long been aware that in both face-to-face interactions and in computer-mediated communication, individuals are influenced by collaborators' spatial proximity (Kraut, Fussell, Brennan \& Siegel, 2002; Peña, Walther \& Hancock, 2007). Decades ago, Cairncross (1997) proposed that mediated communication would be the "death of distance," allowing collaboration to occur independently of spatial proximity. However, most social media connections are still with people who are physically nearby (Bailey, Cao, Kuchler, Stroebel \& Wong, 2018). Social media connections often arise from pre-existing relationships (whether recent or historical), but can also begin virtually. As interactions with others arising initially through social media become more common, we ask, how do virtual interactions with a person who is far away affect other perceptions about the world? In this paper, we use two types of computer-mediated communication (text and virtual reality) to investigate how physical distance and social closeness may interact with proximity to affect perceptions of distant locations, following Won, Shriram and Tamir (2017).

While distance remains a salient cue, communicating through media can change perceptions of others, including perceived social closeness (Andrade, 2014).

Perceptions of social closeness can then in turn change people's perceptions of spatial proximity. In a three-study paper, Won et al. (2017) demonstrated an effect of social closeness on distance perception, such that participants texting with conversational partners whom they believed to be located in a distant city estimated smaller distances to that city the more social closeness they reported. The authors initially explained this

Suggested Citation (APA): Won, A.S., and Kim, B., (2021, October) Effects of Physical Distance and Social Closeness in Virtual Reality and Text on Spatial Distance Estimation. Paper presented at AoIR 2021: The 22nd Annual Conference of the Association of Internet Researchers. Virtual Event: AolR. Retrieved from http:// spir.aoir.org. 
effect with construal theory, but in their discussion proposed an alternative theorymotivated perception. These different theories have different implications for how mediated social connections affect perceptions of the larger world.

Construal theory suggests that psychological distance is conflated across a number of dimensions (Trope \& Liberman, 2010). When people feel socially close to their conversational partners, they might extend this association to other kinds of distance, for example, underestimating the physical distance to the location of their conversational partner. However, motivated perception could also explain this effect. The more of an approach orientation a person has towards their conversational partner, the closer they may estimate them to be (Balcetis, 2016). One way to better understand why this phenomenon occurs is to examine what happens when two people converse who are co-located. If people are simply underestimating distances to locations associated with their conversational partner, then distances to the discussed locations should decrease regardless of where the conversational partners are located. But, if people are mentally shrinking the distance between themselves and their conversational partners, then this effect should not appear when the two people are already in the same location; but only when they are separated.

Thus, we hypothesized that the effect of social distance on the estimated spatial distance to the target city will depend on the presumed location of the conversational partner. We also investigated whether manipulating social presence by using a rich medium such as virtual reality versus texting would change the relationship between social distance, conversational partner location, and estimated physical distance participants.

\section{Methods}

To address these questions, we conducted a between-subjects experiment. Participants were randomly assigned to one of four conditions, crossing two factors. The factor of Location depended on whether the participant believed their conversational partner was located in an adjacent room ("Near" condition) or in another state ("Far" condition). The factor of Medium depended on whether participants were communicating using textbased chat ("Text") or wearing headsets, holding hand controllers, and interacting as avatars in an immersive virtual reality environment ("VR").

Eighty-three participants signed informed consent and were told by the researcher that they would be chatting with another student, either in virtual reality or by text.

Depending on the assigned condition, the researcher either described the other student as enrolled at a collaborating university in Twin Falls, Idaho and connecting remotely (Far condition), or enrolled in summer school at the same university and seated in the lab room next door (Near condition). In fact, all participants spoke with a research assistant confederate, who was located either next door, or in another part of the building. Participants in the VR conditions conversed using a networked virtual reality platform which allowed users to interact using avatars whose movements followed the tracked movements of the users' heads and hands. Participants in the Text conditions used Google Hangout (hangouts.google.com) to communicate. RACs in the near condition described Twin Falls as their home town. RACs in the far condition described 
Twin Falls similarly, and mentioned that was the city they were currently located in. After the interaction, participants filled out a survey rating their sense of social closeness with their conversational partner, as well as their distance estimates to the cities discussed.

\section{Results and Conclusion}

Our hypothesis was supported: the effect of social closeness on the estimated distance to the target city depended on whether conversational partners are co-located or far away. We found a statistically significant interaction between social distance and the location of the conversational partner $(p<.004)$. Social closeness with a "far" conversational partner who was located in the target city discussed correlated negatively with estimated distances to the target city. When partners were co-located, the correlation was positive-the more social closeness participants reported with colocated partners, the larger distance they estimated to the target city.

This partially supports the explanation of motivated perception-participants who liked their conversational partner wished they were closer. However, the increased distance estimated by participants who were in the same location as their conversational partner remains to be explained. Are participants less motivated to engage with the wider world when their social needs are met?

\section{References}

Andrade, A. D. (2014). From Physical Co-location to Perceived Co-presence: "I feel close to you when I use my mobile". Pacific Asia Journal of the Association for Information Systems, 6(1), 2. doi: 10.17705/1pais.06101

Bailey, M., Cao, R., Kuchler, T., Stroebel, J., \& Wong, A. (2018). Social connectedness: Measurement, determinants, and effects. Journal of Economic Perspectives, 32(3), 259-80. doi: 10.1257/jep.32.3.259

Balcetis, E. (2016). Approach and avoidance as organizing structures for motivated distance perception. Emotion Review, 8(2), 115-128. doi: $10.1177 / 1754073915586225$

Cairncross, F. (1997). The death of distance: How the communications revolution will change our lives. Boston, MA: Harvard Business School Press. ISBN: 0-875848060

Kraut, R. E., Fussell, S. R., Brennan, S. E., \& Siegel, J. (2002). Understanding effects of proximity on collaboration: Implications for technologies to support remote collaborative work. Distributed work, 137-162. https://doi.org/10.7551/mitpress/2464.003.0010

Peña, J., Walther, J. B., \& Hancock, J. T. (2007). Effects of geographic distribution on dominance perceptions in computer-mediated groups. Communication Research, 34(3), 313-331. doi: 10.1177/0093650207300431 
The R Project for Statistical Computing. (n.d.). Retrieved September 24, 2018, from https://www.r-project.org/

Trope, Y., \& Liberman, N. (2010). Construal-level theory of psychological distance. Psychological review, 117(2), 440. doi: 10.1037/a0018963

Won, A. S., Shriram, K., \& Tamir, D. I. (2017). Social distance increases perceived physical distance. Social Psychological and Personality Science, 9(3), 372-380. doi: $10.1177 / 194855061770701$ 